\title{
Study on Correlations for Morpho-Physio Traits of Six Wheat Cultivars
}

Aamir Ali Abro ${ }^{1 *}$, Abdul Ghaffar Khoso ${ }^{2}$, Khalid A. E. Eisawi ${ }^{3}$, Ahmed Elsayed Abdelghany ${ }^{4}$, Maksat Batyrbek $^{5}$, Naveed Yaseen Sial ${ }^{6}$, Saba Ambreen Memon ${ }^{7}$, Rimsha Larik ${ }^{8}$ Muhammad Akhtar ${ }^{9}$, Bhart Malhi ${ }^{10}$, Muhammad Usman Zahoor $^{11}$

${ }^{1}$ Department of Plant Breeding \& Genetics, Sindh Agriculture University Tandojam Sindh Pakistan

${ }^{2}$ College of Plant Protection, Northwest Agriculture \& Forestry University Yangling China

${ }^{3}$ College of Forestry, Northwest A \& F University, Yangling, China

${ }^{4}$ College of Water Resources and Architecture Engineering, Northwest A \& F University, Yangling, China.

${ }^{5}$ Department of Soil Science and Agro-Chemistry Kazakh Research Institute of Agriculture and Plant Growing

${ }^{6}$ Department of Agriculture, University College of Dera Murad Jamali Naseerabad (LUAWMS)-80700, Pakistan.

${ }^{7}$ Deartment of Horticulture, Faculty of Crop Production, Sindh Agriculture University Tandojam Sindh Pakistan

${ }^{8}$ Institiute of Chemistry Shah Abdul Latif University Khairpur

${ }^{9}$ Institute of Crop Sciences, Chinese Academy of Agricultural Sciences, Beijing. P. R. China

${ }^{10}$ Southwest University of Science and Technology

${ }^{11}$ Department of Agronomy, Faculty of Agriculture Sciences \& Technology, Bahaudin Zakariya University, Multan

For correspondence: aamirabrahim@gamil.com

Article Received 07-10-2021, Article Revised 28-12-2021, Article Accepted 10-01-2022

Abstract

Current research was conducted to analyze the coefficient and distribution of direct and indirect influence of yield components and earliness characteristics in wheat genotypes. Both experiments were carried out at the Pot House of the (NIA Nuclear Institute of Agriculture, Tandojam) (Latitude: 25.433. Longitude: 68.533), the experiment was established as a randomized complete block design (RCBD). There were six varieties of wheat, namely IBWSN1010, IBWSN 1025, TD1, ESW9525 Chakwal86 and Khirman, which were used to study the correlation coefficient between morphological and physiological traits. The study was based on fifteen (15) morphologically and physiologically traits that were observed. The analysis of variance showed significant differences between the characteristics of the varieties. The ESW9525 showed better performance for all morphological and physiological traits. The correlation and the coefficient analysis showed that the days to maturity and the days to growth predicted significant positive predictions related to the height of the plant $(\mathrm{cm})$ and the length of the ear. Days to $75 \%$ heading and days to $75 \%$ maturity showed negative and positive effects for osmotic potential, relative water content in $\%$ and total chlorophyll content in\%. Plant height $(\mathrm{cm})$ had negative and positive and significant with tiller plant ${ }^{-1}$. Therefore, in our research trial, variety ESW9525 may be favorable for hybridization in order to produce a promising and drought tolerant wheat cultivar.

Keywords: Correlation, Morphological-Physiological traits, Earliness traits,

\section{Introduction}

Correlation studies are very useful for breeders to improve drought tolerance in the sense that any physiological or yield trait with high heritability could be used as indirect selection criteria to improve yield in arid environments (Jatoi et al., 2011). The correlation and coefficient analysis quantifies the interrelationship of the different components of income and also shows whether the effect is directly reflected in income or other ways can be used to achieve an effect. Wheat yield fluctuates with the environment and genetic interactions. Selection to improve grain yield can only be more effective when genetic material showing variability (Khaliqe et al., 2004; Yasin \& Singh, 2010). Wheat is the most cultivated and consumed food grain in the world (Farooq et al., 2011). With progressive climatic changes, global increasing scarcity of water resources and deteriorating ecological environment strongly influence on the production of wheat (Singh \& Chaudhary, 2006). The continued increase in wheat production could be attributed to the development of various modern technologies in conjunction with potentially highyielding wheat varieties. The analysis of the variability of different traits and the presence of a specific trait with another trait that contributes to crop performance and would be the first priority for a successful breeding program (Mary \& Gopalan, 2006). In the case of wheat genotypes, genetic variability can be estimated on the basis of quantitative characteristics. Scheduled parenting is very important for successful breeding. In crops, changes in anatomical and physio-biochemical characteristics due to resistance to drought could be used to develop new genotypes and achieve better production under conditions of water stress (Martínez et al., 2007). However, understanding and choosing the biochemical and physiological basis of tolerance to water stress in plants is important for breeding the crop (Abro et al., 2019).

\section{Materials and methods}


An experiment was carried out in 2018-2019 growing season at the pot house of Nuclear Institute of Agriculture NIA Tandojam to observe the correlation coefficients of the morphological and physiological characteristics of 6 different varieties of wheat (Triticum aestivum L.), namely IBWSN1010, IBWSN 1025, TD1, ESW9525, Khirman and Chakwal86. The seeds were sown in controlled cemented tanks (size $3 \times 3 \times 1$ cubic meters), filled with sandy loam soil, with three replications in the Random Complete Block Design (RCBD). The data of fifteen physiological and agronomic traits were collected, such as proline content ( $\mu$ mol. G1 fresh weight), glycine betaine ( $\mu$ mol. g1 fresh weight), total soluble sugars ( $\mu$ mol. $g 1$ fresh weight), total chlorophyll (mg g1 fresh weight), nitrate reductase activity ((NRA) ( $\mu$ mol. g1 fresh weight hrl), potassium content $(\mathrm{K}+)(\% \mathrm{~g} 1$ fresh weight), osmotic potential (MPa) together with named agronomic characteristics, plant height $(\mathrm{cm})$, ear length $(\mathrm{cm})$, number of plowed plants1, straw/plant weight $(\mathrm{g})$, 100-grain weight $(\mathrm{g})$, seed index (g), days up to $75 \%$ bearing, days up to $75 \%$ maturity.

Statistical analysis. The recorded data was subjected to analysis of variance to distinguish the superiority of the treatment units, and the LSD (least significant differences) test according to $<0.1$ and $<0.5 \%$ of probability level.

\section{Results and discussion.}

Correlation coefficient (r) analysis: Correlation studies were conducted in pot house experiments to provide selection criteria of genotypes with yield. The results are presented in Table

\section{Correlation analysis:}

Days to $75 \%$ heading: Days to $75 \%$ heading showed significant and positive correlations with days to maturity $\left(\mathrm{r}=0.831^{* *}\right)$, plant height $(\mathrm{r}=0.179 *)$, tillers plant $^{-1}(\mathrm{r}=0.291 *)$ and spike length $(\mathrm{r}=0.332 *)$. However, non-significant correlations were observed with grain weight $\left(\mathrm{r}=0.056^{\mathrm{ns}}\right)$, straw weight $\left(\mathrm{r}=0.101^{\mathrm{ns}}\right)$, whereas non-significant and negative correlations were observed between seed index $\left(\mathrm{r}=-0.040^{\mathrm{ns}}\right)$. Physiological traits were non-significant and negatively correlated with this trait.

Days to $\mathbf{7 5 \%}$ maturity: Days to $75 \%$ maturity showed significant and positive correlations with plant height $\left(\mathrm{r}=-0.005^{*}\right)$, tillers $\operatorname{plant}^{-1}\left(\mathrm{r}=0.187^{*}\right)$, spike length $(0.181 *)$ However, non-significant and positive correlations were observed with grain weight $\mathrm{r}=$ $\left(0.290^{\text {ns }}\right)$, straw weight $\left(r=0.183^{\text {ns }}\right)$, seed index $(r=$ $\left.0.129^{\text {ns }}\right)$. Physiological traits were also observed nonsignificant and negative correlations with proline $(\mathrm{r}=$ $\left.0.013^{\text {ns }}\right)$, osmotic potential $\left(\mathrm{r}=-0.131^{\mathrm{ns}}\right)$, potassium content $\left(r=-0.127^{\text {ns }}\right)$, glycine-betaine $\left(r=-0.072^{\text {ns }}\right)$, total soluble sugars $\left(\mathrm{r}=-0.131^{\mathrm{ns}}\right)$, whereas nitrate reeducates activity $\left(\mathrm{r}=0.104^{\mathrm{ns}}\right)$, total chlorophyll content\% $(\mathrm{r}=$ $\left.0.257^{\text {ns }}\right)$ and relative water content $\left(r=0.169^{\text {ns }}\right)$ were observed non-significant and positive correlate

Plant height: Plant height exhibited significant positive correlations with tillers plant ${ }^{-1}(\mathrm{r}=0.501 *)$, spike length $\left(\mathrm{r}=0.793^{* *}\right)$, straw weight $\left(\mathrm{r}=0.613^{* *}\right)$, seed index $(\mathrm{r}=$ $\left.0.700^{* *}\right)$. However, physiological traits also showed significant and negative correlations with proline content $(\mathrm{r}=-0.294 *)$, osmotic potential $\left(\mathrm{r}=-0.479^{*}\right)$, potassium content $\left(r=-0.332^{*}\right)$, glycine-betaine $\left(r=-0.256^{*}\right)$, total soluble sugars $\left(\mathrm{r}=-0.285^{*}\right)$ and nitrate reeducates activity $\left(\mathrm{r}=-0.038^{\mathrm{ns}}\right)$. However, significant and positive correlations were observed between chlorophyll content $\left(\mathrm{r}=0.391^{*}\right)$ and relative water content $\left(\mathrm{r}=0.561^{* *}\right)$ with plant height.

Tillers plant $^{-1}$ : Tillers plant ${ }^{-1}$ had significant positive correlations with spike length $\left(\mathrm{r}=0.616^{* *}\right)$, straw weight $\left(\mathrm{r}=0.621^{* *}\right)$, seed index $(0.694 * *)$. However, nonsignificant and positive correlations were observed between grain weight $\left(\mathrm{r}=0.040^{\mathrm{ns}}\right)$. Physiological traits also showed significant and negative correlations with proline content $(\mathrm{r}=-0.377 *)$, glycine-betaine $(\mathrm{r}=$ $\left.0.361^{*}\right)$, osmotic potential $(\mathrm{r}=-0.412 *)$, and potassium content $\left(\mathrm{r}=-0.127^{\mathrm{ns}}\right)$. However, significant and positive correlation were observed in relative water content $\left(\mathrm{r}=0.601^{* *}\right)$, total chlorophyll content $\left(\mathrm{r}=0.330^{*}\right)$, whereas nitrate reeducates activity $\left(\mathrm{r}=0.104^{\mathrm{ns}}\right)$ and total soluble sugars $\left(\mathrm{r}=-0.131^{\mathrm{ns}}\right)$ had non-significant correlations with each other.

Spike length: Spike length showed significant positive correlations with straw weight $\left(\mathrm{r}=0.667^{* *}\right)$, seed index $\left(\mathrm{r}=0.725^{* *}\right)$. However, non-significant and positive correlations were observed with grain weight $(\mathrm{r}=$ $\left.0.069^{\text {ns }}\right)$, Physiological traits also observed significant and negative correlations with proline $\left(\mathrm{r}=-0.160^{*}\right)$, glycine-betaine $\left(\mathrm{r}=-0.1774^{\mathrm{ns}}\right)$, osmotic potential ($\left.0.380^{*}\right)$, potassium content $\left(\mathrm{r}=-0.1579^{\mathrm{ns}}\right)$, total soluble sugars $\left(r=-0.117^{\mathrm{ns}}\right)$. However significant and positive correlation were observed in relative water content $(\mathrm{r}=$ $\left.0.667^{* *}\right)$, total chlorophyll content $\%\left(r=0.481^{*}\right)$ and nitrate reeducates activity $\left(\mathrm{r}=0.1549^{\mathrm{ns}}\right)$.

Grain weight: Grain weight showed significant and positive correlations with seed index $\left(\mathrm{r}=0.225^{* *}\right)$. However, non-significant and positive correlations were observed between straw weight $\left(r=0.168^{\text {ns }}\right)$ and grain weight. Physiological traits also had significant and negative correlations with osmotic potential $\left(\mathrm{r}=-0.163^{*}\right)$. However, non-significant and negative correlations were observed between proline content $\left(\mathrm{r}=-0.007^{\mathrm{ns}}\right)$, glycinebetaine $\left(\mathrm{r}=-0.078^{\mathrm{ns}}\right)$, nitrate reeducates activity $(\mathrm{r}=$ $\left.0.063^{\mathrm{ns}}\right)$, total soluble sugars $\left(\mathrm{r}=-0.199^{\mathrm{ns}}\right)$ and total chlorophyll content $\left(\mathrm{r}=-0.083^{\mathrm{ns}}\right)$, whereas relative water content $\left(\mathrm{r}=0.130^{\mathrm{ns}}\right)$ and potassium content $\left(\mathrm{r}=0.026^{\mathrm{ns}}\right)$ had non-significant and positive correlation with grain weig 
Table. 1. Correlation coefficient (r) among various morphological and physiological traits of wheat genotypes.

\begin{tabular}{|c|c|c|c|c|c|c|c|c|c|c|c|c|c|c|c|c|}
\hline & $\begin{array}{l}\text { Days } \\
\text { to } \\
\text { heading } \\
\end{array}$ & $\begin{array}{l}\text { Days } \\
\text { To } \\
\text { maturity }\end{array}$ & $\begin{array}{l}\text { Plant } \\
\text { height }\end{array}$ & $\begin{array}{l}\text { Tillers } \\
\text { plant }^{-1}\end{array}$ & $\begin{array}{l}\text { Spike } \\
\text { length }\end{array}$ & $\begin{array}{l}\text { Grain } \\
\text { weight }\end{array}$ & $\begin{array}{l}\text { Straw } \\
\text { weight }\end{array}$ & $\begin{array}{l}\text { Seed } \\
\text { index }\end{array}$ & $\begin{array}{l}\text { Proline } \\
\text { content }\end{array}$ & $\begin{array}{l}\text { Osmotic } \\
\text { potential }\end{array}$ & $\begin{array}{l}\text { Relative } \\
\text { water } \\
\text { content }\end{array}$ & $\begin{array}{l}\text { Potassiu } \\
\text { m }\end{array}$ & $\begin{array}{l}\text { Glycin } \\
\text { e }\end{array}$ & $\begin{array}{l}\text { Nitrate } \\
\text { reeducate } \\
\text { s activity } \\
\end{array}$ & $\begin{array}{l}\text { Total } \\
\text { sugar }\end{array}$ & $\begin{array}{l}\text { Total } \\
\text { chlorophy } \\
\text { ll }\end{array}$ \\
\hline Days to heading & - & & & & & & & & & & & & & & & \\
\hline Days to maturity & $0.830^{* *}$ & - & & & & & & & & & & & & & & \\
\hline Plant height & $0.179 *$ & $0.152 *$ & - & & & & & & & & & & & & & \\
\hline Tillers plant-1 & $0.291 *$ & $0.187^{*}$ & $0.501^{*}$ & - & & & & & & & & & & & & \\
\hline Spike length & $0.332^{*}$ & $0.181^{*}$ & $0.793 * *$ & $0.616^{* *}$ & - & & & & & & & & & & & \\
\hline Grain weight & $0.056^{\mathrm{ns}}$ & $0.290^{\mathrm{ns}}$ & $0.101^{\mathrm{ns}}$ & $0.040^{\mathrm{ns}}$ & $0.069^{\mathrm{ns}}$ & - & & & & & & & & & & \\
\hline Straw weight & $0.101^{\text {ns }}$ & $0.181^{\text {ns }}$ & $0.613^{* *}$ & $0.621^{* *}$ & $0.667 * *$ & $0.1683^{\text {ns }}$ & - & & & & & & & & & \\
\hline Seed Index & $-0.040^{\mathrm{ns}}$ & $0.129^{\mathrm{ns}}$ & $0.700^{* *}$ & $0.694 * *$ & 0.725 ** & $0.225^{* *}$ & $0.693 * *$ & - & & & & & & & & \\
\hline Proline content & $0.011^{\mathrm{ns}}$ & $-0.013^{\text {ns }}$ & $-0.294 *$ & $-0.377^{*}$ & $-0.160 *$ & $-0.000^{\mathrm{ns}}$ & $-0.669 * *$ & $-0.421 *$ & - & & & & & & & \\
\hline Osmotic potential & $-0.029^{\mathrm{ns}}$ & $-0.131^{\mathrm{ns}}$ & $-0.479 *$ & $-0.412^{*}$ & $-0.380 *$ & $-0.163^{*}$ & $-0.794 * *$ & $-0.655^{* *}$ & $0.817 * *$ & - & & & & & & \\
\hline $\begin{array}{l}\text { Relative water } \\
\text { content }\end{array}$ & $-0.084^{\mathrm{ns}}$ & $0.169^{*}$ & $0.561 * *$ & $0.601 * *$ & $0.667^{* * *}$ & $0.130^{\mathrm{ns}}$ & $0.546^{* *}$ & $0.789 * *$ & $-0.164 *$ & $-0.369 *$ & - & & & & & \\
\hline Potassium & $-0.021^{\mathrm{ns}}$ & $-0.127^{\mathrm{ns}}$ & $-0.332^{*}$ & $-0.131^{\mathrm{ns}}$ & $-0.157^{\mathrm{ns}}$ & $0.026^{\mathrm{ns}}$ & $-0.425 * *$ & $-0.299 * *$ & $0.670^{* * *}$ & $0.675 * *$ & $-0.061^{\mathrm{ns}}$ & - & & & & \\
\hline Glycine-betain & $-0.021^{\mathrm{ns}}$ & $-0.072^{\mathrm{ns}}$ & $-0.256^{*}$ & $-0.361 *$ & $-0.177^{\text {ns }}$ & $-0.078^{\mathrm{ns}}$ & $-0.699 * *$ & $-0.460 * *$ & $0.960 * *$ & $0.893^{* *}$ & $-0.177 *$ & $0.658^{* * *}$ & - & & & \\
\hline $\begin{array}{l}\text { Nitrate reducates } \\
\text { activity }\end{array}$ & $-0.086^{\mathrm{ns}}$ & $0.104^{\mathrm{ns}}$ & $-0.038^{\text {ns }}$ & $0.159^{\text {ns }}$ & $0.154^{\mathrm{ns}}$ & $-0.063^{\mathrm{ns}}$ & $0.187^{\mathrm{ns}}$ & $0.339 *$ & $-0.300^{*}$ & $-0.298^{*}$ & $0.283^{*}$ & $-0.247 *$ & $-0.324 *$ & - & & \\
\hline Total sugar & $-0.042^{\mathrm{ns}}$ & $-0.131^{\mathrm{ns}}$ & $-0.285^{*}$ & $-0.039^{\text {ns }}$ & $-0.117^{\mathrm{ns}}$ & $-0.199^{\text {ns }}$ & $-0.310^{*}$ & $-0.400^{*}$ & $0.292 *$ & $0.540^{* *}$ & $-0.301 * *$ & $0.419^{* * *}$ & $0.429 * *$ & $-0.231 *$ & - & \\
\hline Total chlorophyll & $0.234^{\mathrm{ns}}$ & $0.257^{\mathrm{ns}}$ & $0.391 *$ & $0.330^{*}$ & $0.481 *$ & $-0.083^{\text {ns }}$ & $0.183^{\text {ns }}$ & $0.424 * *$ & $0.075^{\mathrm{ns}}$ & $-0.051^{\mathrm{ns}}$ & $0.422 * *$ & $0.023^{\text {ns }}$ & $0.050^{\mathrm{ns}}$ & $0.335^{*}$ & $\begin{array}{l}- \\
0.211^{*}\end{array}$ & - \\
\hline
\end{tabular}

$* *=$ significant at $\mathbf{P}<0.01$ probability level, *= Significant at $\mathbf{P}<0.05$ probability level and $\mathbf{n s =}$ Non-significant 
Table. 2 (a). Mean value of Correlation coefficient (r) among various morphological traits of wheat genotypes.

\begin{tabular}{|c|c|c|c|c|c|c|c|c|c|c|c|c|}
\hline \multirow{2}{*}{ Genotypes } & \multicolumn{3}{|c|}{ Days to heading } & \multicolumn{3}{|c|}{ Days to Maturity } & \multicolumn{3}{|c|}{ Plant Height } & \multicolumn{3}{|c|}{ Tillers plant-1 } \\
\hline & Control & Drought & Mean & Control & Drought & Mean & Control & Drought & Mean & Control & Drought & Mean \\
\hline IBWSN-1010 & 80.7 & 78.5 & $79.6 \mathrm{a}$ & 120.7 & 116.16 & $118.43 \mathrm{a}$ & 79.7 & 61.3 & $70.50 \mathrm{~b}$ & 8 & 5 & $6.50 \mathrm{~b}$ \\
\hline IBWSN-1025 & 79.5 & 76.4 & $77.95 \mathrm{~b}$ & 117.8 & 114.61 & $116.20 \mathrm{ab}$ & 70.3 & 57.3 & $63.83 \mathrm{c}$ & 11 & 8 & $9.50 \mathrm{a}$ \\
\hline TD-1 & 60.3 & 56.5 & $58.4 \mathrm{f}$ & 110.3 & 107.06 & $108.68 \mathrm{~d}$ & 52.7 & 46.7 & $49.67 \mathrm{c}$ & 11 & 8 & $9.50 \mathrm{a}$ \\
\hline ESW-9525 & 77.2 & 72.4 & $74.8 \mathrm{c}$ & 115.1 & 111.75 & $113.42 \mathrm{c}$ & 72.7 & 66.3 & $69.50 \mathrm{~b}$ & 12 & 10 & $11.00 \mathrm{a}$ \\
\hline Khirman & 74.3 & 70.5 & $72.4 \mathrm{~d}$ & 114.5 & 113.85 & $114.17 \mathrm{bc}$ & 91.3 & 69.7 & $80.50 \mathrm{a}$ & 12 & 10 & $11.00 \mathrm{a}$ \\
\hline Chakwal-86 & 68.4 & 65.5 & $66.95 \mathrm{e}$ & 115.4 & 113.3 & $114.35 \mathrm{bc}$ & 81 & 63 & $72.00 \mathrm{~b}$ & 12 & 10 & $11.00 \mathrm{a}$ \\
\hline Mean & $73.4 \mathrm{a}$ & $69.96 \mathrm{~b}$ & & $115.63 \mathrm{a}$ & $112.79 \mathrm{~b}$ & & $74.6 \mathrm{a}$ & $60.7 \mathrm{~b}$ & & $11.0 \mathrm{a}$ & $8.5 \mathrm{~b}$ & \\
\hline LSD & & & 0.05 & & & 0.05 & & & 0.05 & & & 0.05 \\
\hline Treatments & & & 35.363 & & & 24.28 & & & 3.0518 & & & 1.4928 \\
\hline Genotypes & & & 124.409 & & & 21.2 & & & 4.4667 & & & 1.5392 \\
\hline $\begin{array}{c}\text { Treatments X } \\
\text { Genotypes }\end{array}$ & & & 116.892 & & & 2.4081 & & & 6.3168 & & & 2.1767 \\
\hline $\mathrm{CV} \%$ & & & 1.6366 & & & 0.82 & & & 5.48 & & & 13.11 \\
\hline
\end{tabular}

Straw weight: Straw weight showed significant positive correlations with seed index $\left(\mathrm{r}=0.693^{* *}\right)$. Physiological traits also observed significant and negative correlations with proline $\left(-0.669^{* *}\right)$, osmotic potential $(\mathrm{r}=-0.794 * *)$, potassium content $(\mathrm{r}=$ $\left.0.4251^{* *}\right)$, glycine-betaine $\left(\mathrm{r}=-0.699^{* *}\right)$, total soluble sugars $(\mathrm{r}=-0.310 *)$. However, non-significant and positive correlations were observed in total chlorophyll content $\left(\mathrm{r}=0.2576^{\mathrm{ns}}\right)$ and nitrate reeducates activity $(\mathrm{r}=$ $\left.0.187^{\mathrm{ns}}\right)$, whereas relative water content $\left(\mathrm{r}=0.546^{* *}\right)$ showed significant and positive correlations with straw weight.

Seed index: Seed index exhibited non-significant negative associations with physiological traits such as proline $\left(\mathrm{r}=-0.0135^{\mathrm{ns}}\right)$, osmotic potential $\left(\mathrm{r}=-0.131^{\mathrm{ns}}\right)$, potassium content $\left(\mathrm{r}=-0.127^{\mathrm{ns}}\right)$, total soluble sugars $(\mathrm{r}=$ $\left.-0.131^{\mathrm{ns}}\right)$ glycine-betaine $\left(\mathrm{r}=-0.072^{\mathrm{n}} \mathrm{s}\right)$, whereas relative water content $\left(\mathrm{r}=0.169^{\mathrm{ns}}\right)$, nitrate reeducates activity $\left(\mathrm{r}=0.104^{\mathrm{ns}}\right)$, and total chlorophyll content\% ( $\mathrm{r}=$ $\left.0.257^{\mathrm{ns}}\right)$ were observed non-significant and positive correlate.

\section{Correlations of physiological traits:}

Proline: Proline revealed significant and positive correlations with osmotic potential $\left(\mathrm{r}=0.817^{* *}\right)$, potassium content $\left(\mathrm{r}=0.670^{* *}\right)$, glycine-betaine $(\mathrm{r}=$ $0.960 * *)$, total soluble sugars $(\mathrm{r}=0.292 *)$ and total chlorophyll content\% $\left(0.075^{*}\right)$. However significant and negative correlation were observed in relative water content $(\mathrm{r}=-0.164 *)$ and nitrate reductase activity $(\mathrm{r}=-0.300 *)$,

Osmotic potential: Osmotic potential showed significant and positive correlations were observed with potassium content $\left(\mathrm{r}=0.675^{* *}\right)$, glycine-betaine $\left(\mathrm{r}=0.893^{* *}\right)$ and total soluble sugars $(\mathrm{r}=0.540 * *)$. However, significant and negative correlations with relative water content $\left(\mathrm{r}=-0.369^{*}\right)$, nitrate reeducates activity $\left(-0.298^{*}\right)$ and total chlorophyll content\% ($\left.0.051^{\mathrm{ns}}\right)$.

Relative water content: Relative water content depicted significant negative correlations with glycinebetaine $(\mathrm{r}=-0.177 *)$, total soluble sugars $(\mathrm{r}=-0.301 *)$ and potassium content $\left(\mathrm{r}=-0.061^{\mathrm{ns}}\right)$. However, significant and positive correlations were observed with total chlorophyll content $\%\left(r=0.422^{* *}\right)$ and nitrate reeducates activity $\left(\mathrm{r}=0.283^{*}\right)$,

Potassium content: Potassium content exhibited significant positive correlations with glycine-betaine $\left(\mathrm{r}=0.658^{* *}\right)$ and total soluble sugars $(\mathrm{r}=0.419 * *)$. However, it had non-significant and positive correlations with nitrate reeducates activity $(\mathrm{r}=$ $\left.0.247^{*}\right)$, whereas total chlorophyll content\% ( $\mathrm{r}=$ $0.023^{\text {ns }}$ ) observed non-significant and positive correlate. 
Table. 2 (b). Mean value of Correlation coefficient (r) among various morphological traits of wheat genotypes.

\begin{tabular}{|c|c|c|c|c|c|c|c|c|c|c|c|c|}
\hline \multirow[b]{2}{*}{ Genotypes } & \multicolumn{3}{|c|}{ Spike length } & \multicolumn{3}{|c|}{ Grain weight } & \multicolumn{3}{|c|}{ Straw weight } & \multicolumn{3}{|c|}{ Seed index } \\
\hline & Control & Drought & Mean & Control & Drought & Mean & Control & Drought & Mean & Control & Drought & Mean \\
\hline IBWSN-1010 & 11.9 & 9.3 & $10.59 \mathrm{c}$ & 4.6 & 14.8 & $9.70 \mathrm{~d}$ & 20.3 & 8.4 & $14.33 \mathrm{~d}$ & 46.1 & 37.8 & $41.9 \mathrm{a}$ \\
\hline IBWSN-1025 & 12.5 & 9.8 & $11.11 \mathrm{c}$ & 22.2 & 18.7 & $20.42 \mathrm{a}$ & 25.1 & 9.2 & $17.16 \mathrm{~cd}$ & 43.9 & 34.7 & $\begin{array}{c}39.3 \\
\mathrm{ab}\end{array}$ \\
\hline TD-1 & 10.8 & 7.6 & $9.18 \mathrm{~d}$ & 19.7 & 15.2 & $17.46 \mathrm{c}$ & 26.2 & 10.3 & $18.29 \mathrm{~cd}$ & 41.6 & 29.1 & $35.4 \mathrm{~b}$ \\
\hline ESW-9525 & 14.7 & 13.2 & $13.98 \mathrm{a}$ & 23.3 & 19.7 & $21.48 \mathrm{a}$ & 39.1 & 14.3 & $26.71 \mathrm{a}$ & 46.2 & 39.4 & $42.8 \mathrm{a}$ \\
\hline Khirman & 14.7 & 12.1 & $13.40 \mathrm{ab}$ & 23.4 & 19.2 & $21.31 \mathrm{a}$ & 36.2 & 13.7 & $24.94 \mathrm{ab}$ & 45.7 & 37.7 & $41.7 \mathrm{a}$ \\
\hline Chakwal-86 & 13.8 & 11.3 & $12.58 \mathrm{~b}$ & 21.3 & 17.2 & $\begin{array}{c}19.22 \\
\mathrm{ab}\end{array}$ & 30.3 & 11.2 & $20.7 \mathrm{bc}$ & 45.5 & 37.3 & $41.4 \mathrm{a}$ \\
\hline Mean & $13.1 \mathrm{a}$ & $10.6 \mathrm{~b}$ & & $19.1 \mathrm{a}$ & $17.5 \mathrm{~b}$ & & $29.5 \mathrm{a}$ & $11.2 \mathrm{~b}$ & & $44.8 \mathrm{a}$ & $36.0 \mathrm{~b}$ & \\
\hline LSD & & & 0.05 & & & 0.05 & & & 0.05 & & & 0.05 \\
\hline Treatments & & & 2.0348 & & & 12.627 & & & 5.0545 & & & 3.3362 \\
\hline Genotypes & & & 1.1275 & & & 13.16 & & & 4.3686 & & & 4.3634 \\
\hline $\begin{array}{c}\text { Treatments X } \\
\text { Genotypes }\end{array}$ & & & 1.5946 & & & 18.611 & & & 6.1782 & & & 6.1708 \\
\hline $\mathrm{CV} \%$ & & & 7.93 & & & 51.44 & & & 17.82 & & & 8.97 \\
\hline
\end{tabular}

Table. 2 (c). Mean value of Correlation coefficient (r) among various physiological traits of wheat genotypes.

\begin{tabular}{|c|c|c|c|c|c|c|c|c|c|c|c|c|}
\hline \multirow[b]{2}{*}{ Genotypes } & \multicolumn{3}{|c|}{ Proline content } & \multicolumn{3}{|c|}{ Osmotic potential } & \multicolumn{3}{|c|}{ Relative water content } & \multicolumn{3}{|c|}{ Potassium } \\
\hline & Control & Drought & Mean & Control & Drought & Mean & Control & Drought & Mean & Control & Drought & Mean \\
\hline IBWSN-1010 & 8.46 & 76.02 & $42.24 \mathrm{c}$ & 0.58 & 0.91 & $0.75 \mathrm{~d}$ & 76.37 & 55.46 & $\begin{array}{c}65.92 \\
\mathrm{a}\end{array}$ & 2.17 & 2.27 & $2.22 \mathrm{c}$ \\
\hline IBWSN-1025 & 13.35 & 86.03 & $49.69 \mathrm{~b}$ & 0.59 & 1.1 & $0.85 \mathrm{c}$ & 77.7 & 55.71 & $\begin{array}{c}66.71 \\
\mathrm{a}\end{array}$ & 2.52 & 3.24 & $2.88 \mathrm{~b}$ \\
\hline TD-1 & 4.62 & 7.72 & $6.17 \mathrm{~d}$ & 0.8 & 1.16 & $0.98 \mathrm{~b}$ & 70.17 & 34.69 & $\begin{array}{c}52.43 \\
\mathrm{~b}\end{array}$ & 2.24 & 3.17 & $2.71 \mathrm{~b}$ \\
\hline ESW-9525 & 28.03 & 95.14 & $61.59 \mathrm{a}$ & 0.7 & 1.34 & $1.02 \mathrm{a}$ & 80.21 & 68.86 & $\begin{array}{c}74.54 \\
\mathrm{a}\end{array}$ & 3.1 & 3.69 & $3.40 \mathrm{a}$ \\
\hline Khirman & 9.08 & 77.82 & $43.45 \mathrm{c}$ & 0.71 & 1.35 & $1.03 \mathrm{a}$ & 76.55 & 67.06 & $\begin{array}{c}71.80 \\
\mathrm{a}\end{array}$ & 2.63 & 3.11 & $2.87 \mathrm{~b}$ \\
\hline Chakwal-86 & 8.8 & 77.61 & $43.21 \mathrm{c}$ & 0.72 & 1.33 & $1.03 \mathrm{a}$ & 76.92 & 67.65 & $\begin{array}{c}72.28 \\
a\end{array}$ & 2.45 & 3.27 & $2.86 \mathrm{~b}$ \\
\hline Mean & $12.06 \mathrm{~b}$ & $70.06 \mathrm{a}$ & & $0.68 \mathrm{~b}$ & $1.20 \mathrm{a}$ & & $76.32 \mathrm{a}$ & $58.24 \mathrm{~b}$ & & $2.52 \mathrm{~b}$ & $3.13 \mathrm{a}$ & \\
\hline LSD & & & 0.05 & & & 0.05 & & & 0.05 & & & 0.05 \\
\hline Treatments & & & 6.4424 & & & 140.19 & & & 12.409 & & & 0.531 \\
\hline Genotypes & & & 3.6089 & & & 117.71 & & & 12.343 & & & 0.266 \\
\hline $\begin{array}{c}\text { Treatments } X \\
\text { Genotypes }\end{array}$ & & & 5.1038 & & & 166.47 & & & 17.456 & & & 0.3762 \\
\hline CV \% & & & 7.3 & & & 567.25 & & & 15.23 & & & 7.83 \\
\hline
\end{tabular}

Glycine-betaine content: Glycine-betaine content depicted significant and negative correlations with nitrate reductase activity $(\mathrm{r}=-0.324 *)$, However, nonsignificant and positive correlations were observed with total chlorophyll content $\left(\mathrm{r}=0.050^{\mathrm{ns}}\right)$, whereas total soluble sugars $\mathrm{r}=\left(0.429^{* *}\right)$ observed significant and positive correlate. 
Nitrate reductase activity: Nitrate reductase activity showed significant negative correlations with total soluble sugars $\left(r=-0.231^{*}\right)$. However, significant and positive correlations were observed with total chlorophyll content $\left(\mathrm{r}=0.335^{*}\right)$.

Total soluble sugar: Total soluble sugar revealed onsignificant and negative associations with observed total chlorophyll content\% $\left(\mathrm{r}=-0.211^{\mathrm{ns}}\right)$.
Chlorophyll content (mg $\mathbf{g}^{-1}$ fresh wt.): Total chlorophyll content remains highly significant correlated with relative water content, seed index and significant for plant height, tillers per plant, spike length and nitrate reductase activity

Table. 2(d). Mean value of Correlation coefficient ( $r$ ) among various physiological traits of wheat genotypes.

\begin{tabular}{|c|c|c|c|c|c|c|c|c|c|c|c|c|}
\hline \multirow[b]{2}{*}{ Genotypes } & \multicolumn{3}{|c|}{ Glycine } & \multicolumn{3}{|c|}{ Nitrate reducates activity } & \multicolumn{3}{|c|}{ Total sugar } & \multicolumn{3}{|c|}{ Total chlorophyll } \\
\hline & Control & Drought & Mean & Control & Drought & Mean & Control & Drought & Mean & Control & Drought & Mean \\
\hline IBWSN-1010 & 20.22 & 39.86 & $30.04 \mathrm{~b}$ & 0.29 & 0.11 & $0.20 \mathrm{a}$ & 0.76 & 0.8 & $0.78 \mathrm{~b}$ & 0.83 & 0.45 & $0.64 \mathrm{~b}$ \\
\hline IBWSN-1025 & 12.36 & 42.01 & $27.19 \mathrm{c}$ & 0.2 & 0.13 & $\begin{array}{c}0.16 \\
\mathrm{bc}\end{array}$ & 0.73 & 0.83 & $0.78 \mathrm{~b}$ & 0.26 & 0.16 & $0.21 \mathrm{~d}$ \\
\hline TD-1 & 12.83 & 19.62 & $16.23 \mathrm{~d}$ & 0.25 & 0.16 & $0.20 \mathrm{ab}$ & 0.97 & 1.44 & $1.21 \mathrm{a}$ & 0.28 & 0.13 & $0.21 \mathrm{~d}$ \\
\hline ESW-9525 & 19.11 & 54.89 & $37.00 \mathrm{a}$ & 0.2 & 0.2 & $0.20 \mathrm{a}$ & 1.12 & 1.28 & $1.20 \mathrm{a}$ & 0.72 & 0.71 & $0.71 \mathrm{~b}$ \\
\hline Khirman & 18.12 & 56.41 & $37.27 \mathrm{a}$ & 0.14 & 0.14 & $0.14 \mathrm{c}$ & 0.98 & 1.44 & $1.21 \mathrm{a}$ & 0.43 & 0.36 & $0.39 \mathrm{c}$ \\
\hline Chakwal-86 & 15.11 & 48.51 & $31.81 \mathrm{~b}$ & 0.19 & 0.17 & $0.18 \mathrm{ab}$ & 0.85 & 0.99 & $0.92 \mathrm{~b}$ & 0.92 & 0.82 & $0.87 \mathrm{a}$ \\
\hline Mean & $16.29 \mathrm{~b}$ & $43.55 \mathrm{a}$ & & $0.21 \mathrm{a}$ & $0.15 \mathrm{~b}$ & & $0.90 \mathrm{~b}$ & $1.13 \mathrm{a}$ & & $0.57 \mathrm{a}$ & $0.44 \mathrm{~b}$ & \\
\hline LSD & & & 0.05 & LSD & & 0.05 & LSD & & 0.05 & LSD & & 0.05 \\
\hline Treatments & & & 1.4536 & Treatments & & 0.017 & Treatments & & 0.2521 & Treatments & & 0.0984 \\
\hline Genotypes & & & 1.9279 & Genotypes & & 0.0282 & Genotypes & & 0.1897 & Genotypes & & 0.0946 \\
\hline $\begin{array}{c}\text { Treatments X } \\
\text { Genotypes }\end{array}$ & & & 2.7264 & $\begin{array}{c}\text { Treatments } \\
\text { X } \\
\text { Genotypes } \\
\end{array}$ & & 0.0398 & $\begin{array}{c}\text { Treatments } \\
\text { X } \\
\text { Genotypes }\end{array}$ & & 0.2683 & $\begin{array}{c}\text { Treatments } \\
\text { X } \\
\text { Genotypes } \\
\end{array}$ & & 0.1338 \\
\hline CV \% & & & 5.35 & $\mathrm{CV} \%$ & & 12.9 & $\mathrm{CV} \%$ & & 15.52 & $\mathrm{CV} \%$ & & 15.56 \\
\hline
\end{tabular}

\section{Discussion}

The study of drought was one of the complex global physical and chemical processes in which severity occurs in the growth stage of plants (Khakwani et al., 2011). Correlation studies are essential to measure size and directional relationship with grain yield and related traits (Rajper et al., 2018). The correlation coefficient of yield and related traits, indicating an inherent association between the traits, is shown in Table 1. The results showed that the days to maturity and the days to growth predicted a significantly positive association with the height of the plant $(\mathrm{cm})$ and the length of the ear $(\mathrm{cm})$. (Nemat et al., 2015), also predicted a significant and positive correlation, which reflects that plant height $(\mathrm{cm})$ and ear length $(\mathrm{cm})$ will eventually increase with increasing days of sprouting and maturity. However, negative and positive associations were observed with grain weight (g), straw weight (g) and seed index (g), days at $75 \%$ forcing and days at $75 \%$ maturity, content Relative water\%, potassium content, glycine betaine, nitrate reductase activity, plus total soluble sugar and \% total chlorophyll. Similar results with a significant correlation with other detection traits and genotypes were also examined for other breeding programs (Habibpor et al., 2011). A negative correlation was also often found between performance under stress conditions and flowering date (Dodig et al., 2016). The grain yield of a variety depends on its yield components, since (Sheron et al., 1986) observed that plant height, ear length and kernels per ear are directly related to grain yield. Similarly (Shamsuddin, 1987) reported that ears per plant, kernels per ear, 1000 kernel weight, crop index, glume weight, and biological yield are directly related to wheat grain yield. According to another study of the components of durum wheat yield (Simane, 1993). The height of the plant $(\mathrm{cm})$, the tiller plant-1 had negative and positive and significant and non-significant results for the characteristics tiller plant ${ }^{-1}$, ear length, grain weight, straw weight and seed index. In dry tests, a positive and significant correlation was observed between grain yield and grain filling time, chlorophyll content, number of grains per ear, and ear weight. However, a negative and significant correlation was observed between grain yield and the number of days to drive (Hasan Kilic and Yagbasanlar, 2010). However, the association with physiological characteristics showed a 
negative and positive correlation with proline content, osmotic potential, relative water content, Potassium content, glycine betaine, nitrate reductase activity, total soluble sugar and total chlorophyll content. The length of the ear and the weight of the grain showed significant positive correlations with the seed index. However, both traits did not observe significant and positive correlations with grain weight. The observed physiological characteristics, negligible and negative correlations, were observed with proline, glycine betaine, nitrate remodeling activity, total soluble sugars and total chlorophyll content. The straw weight showed significant positive correlations with the seed index. The proline content had a significant and positive association with the osmotic potential, the potassium content, the betaine glycine, the total soluble sugar and the total chlorophyll content. However, in terms of relative water content, significant and negative correlations were observed with nitrate reductase activity. The proline content increases significantly under stress conditions. The positive correlation observed between grain yield and proline content under drought stress conditions shows that ultimately proline accumulation could be seen as a tool for effective selection of drought-tolerant genotypes (Mwadzingeni et al., 2016). The relative water content showed significant negative correlations with glycine betaine, total soluble sugars and potassium content. Potassium and glycine betaine showed significant and negative correlations with nitrate reductase activity. Total soluble sugar showed significant and negative associations with the total chlorophyll content $\%$.

\section{Conclusion}

From the present study it was concluded that the ESW9525 showed better performance in all morphological and physiological traits, the correlation and the coefficient showed that the days to maturity and the days to growth predicted significant positive predictions related to the height of the plant $(\mathrm{cm})$ and the length of the ear. Days to $75 \%$ heading and Days to $75 \%$ maturity showed negative and positive effects respectively osmotic potential, relative water content in $\%$ and total chlorophyll content in \%. Plant height (cm) had negative and positive and significant with tiller plant ${ }^{-1}$. Therefore, variety ESW9525 may be favorable for hybridization in order to produce a promising and drought tolerant wheat cultivar.

\section{Reference}

Ali, M. A., Nawab, N. N., Rasool, G. H. U. L. A. M., \& Saleem, M. U. H. A. M. M. A. D. (2008). Estimates of variability and correlations for quantitative traits in Cicer arietinum. J. Agric. Soc. Sci, 4: 177-179.

Dodig, D., Savić, J., Kandić, V., Zorić, M., RADOVIĆ, B. V., Popović, A., \& Quarrie, S. (2016). Responses of wheat plants under post-anthesis stress induced by defoliation: I. Contribution of agro-physiological traits to grain yield. Experimental Agriculture, 52:(2): 203-223.

Farooq, J., Khaliq, I., Ali, M. A., Kashif, M., Rehman, A. U., Naveed, M., \& Farooq, A. (2011). Inheritance pattern of yield attributes in spring wheat at grain filling stage under different temperature regimes. Australian Journal of Crop Science, 5(13): 1745-1753.

Gomez, K. A., \& Gomez, A. A. (1984). Statistical procedures for agricultural research. John Wiley \& Sons.

Habibpor, M., Valizadeh, M., Shahbazi, H., \& Ahmadizadeh, M. (2011). Study of drought tolerance with cell membrane stability testing and relation with the drought tolerance indices in genotypes of wheat (Triticum aestivum L.). World Applied Sciences Journal, 13(7): 16541660.

Jaleel, C. A., Manivannan, P. A. R. A. M. A. S. I. V. A. M., Wahid, A., Farooq, M., Al-Juburi, H. J., Somasundaram, R. A. M. A. M. U. R. T. H. Y., \& Panneerselvam, R. (2009). Drought stress in plants: a review on morphological characteristics and pigments composition. Int. J. Agric. Biol, 11(1): 100-105.

Jatoi, W. A., Baloch, M. J., Kumbhar, M. B., Khan, N. U., \& Kerio, M. I. (2011). Effect of water stress on physiological and yield parameters at anthesis stage in elite spring wheat cultivars. Sarhad J. Agric, 27(1): 59-65.

Khakwani, A. A., Dennett, M. D., \& Munir, M. (2011). Early growth response of six wheat varieties under artificial osmotic stress condition. Pak. J. Agric. Sci, 48: 121-126.

Martínez, I. G., Prat, C., Ovalle, C., del Pozo, A., Stolpe, N., \& Zagal, E. (2012). Subsoiling improves conservation tillage in cereal production of severely degraded Alfisols under Mediterranean climate. Geoderma, 189: 10-17.

Mary, S. S \& Gopalan, A. (2006). Dissection of genetic attributes yield traits of fodder cowpea in F3 and F4. Journal of Applied Sciences Research, 2: 805-808.

Mwadzingeni, L., Shimelis, H., Dube, E., Laing, M. D., \& Tsilo, T. J. (2016). Breeding wheat for drought tolerance: Progress and technologies. Journal of Integrative Agriculture, 15(5): 935943.

Hassan, N. M., El-Bastawisy, Z. M., El-Sayed, A. K., Ebeed, H. T., \& Alla, M. M. N. (2015). Roles of dehydrin genes in wheat tolerance to drought stress. Journal of advanced research, 6(2): 179188.

Rajper, A. A., Baloch, S. K., Baloch, K., Ahmed, S., Kaleri, A. A., Leghari, A. L., \& Kaleri, R. R. 
(2018). 14. Analysis path coefficient of yield earliness traits in wheat (Triticum aestivum L.). Pure and Applied Biology, 7(1): 112-120.

Shamsuddin, A. K. (1987). Path analysis in breadwheat. Indian Journal of Agricultural Sciences, 57: 47-49.

Sheron, Z., Lauthra, O. S \& Kuhad, M. S. (1986). Association of physiological and biochemical character with the yield of rain fed. Plant Breeding, 56, 54-27.

Simane, B. (1993). Drought resistance indurum wheat. ISBN9054851627.See discussions, stats, and author profiles for this publication at
Singh, G. P., \& Chaudhary, H. B. (2006). Selection parameters and yield enhancement of wheat (Triticum aestivum L.) under different moisture stress conditions. Asian Journal of Plant Sciences. 5: 894-898

Soomro, F. A., Mujtaba, S. M., Soomro, A. A., Soomro, A. A., \& Jian, Z. H. A. N. G. (2014). A route-map to the analysis of drought stress tolerance in Wheat (Triticum aestivum L.) genotypes. Eur Acad Res, 2: 12328-38.

Singh, S. (2010). Correlation and path coefficient analyses in sunflower. Journal of Plant Breeding and Crop Science, 2(5):129-133.

Publisher's note: JOARPS remains neutral with regard to jurisdictional claims in published maps and institutional affiliations.

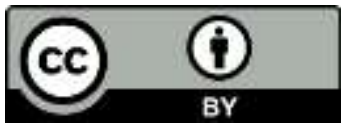

This is an open access article distributed under the terms of the Creative Commons Attribution License (CC BY 4.0), which permits unrestricted use, distribution, and reproduction in any medium, provided the original author and source are credited. To view a copy of this license, visit http://creativecommons.org/licenses/by/4.0/. 